\title{
Laplace pressure as a surface stress in fluid vesicles
}

\author{
Jemal Guven \\ Instituto de Ciencias Nucleares, Universidad Nacional Autónoma de México \\ Apdo. Postal 70-543, 04510 México, DF, MEXICO
}

\begin{abstract}
Consider a surface, enclosing a fixed volume, described by a free-energy depending only on the local geometry; for example, the Canham-Helfrich energy quadratic in the mean curvature describes a fluid membrane. The stress at any point on the surface is determined completely by geometry. In equilibrium, its divergence is proportional to the Laplace pressure, normal to the surface, maintaining the constraint on the volume. It is shown that this source itself can be expressed as the divergence of a positiondependent surface stress. As a consequence, the equilibrium can be described in terms of a conserved effective surface stress. Various non-trivial geometrical consequences of this identification are explored. In a cylindrical geometry, the cross-section can be viewed as a closed planar Euler elastic curve. With respect to an appropriate centre the effective stress itself vanishes; this provides a remarkably simple relationship between the curvature and the position along the loop. In two or higher dimensions, it is shown that the only geometry consistent with the vanishing of the effective stress is spherical. It is argued that the appropriate generalization of the loop result will involve null stresses.
\end{abstract}

August 21, 2018

PACS: 04.60.Ds

\section{Introduction}

The energy of a soap bubble is proportional to its surface area. The bubble is round because this is the shape of the surface, enclosing a fixed volume, which minimizes area [1]. The shape of a closed surface described by an energy which depends in some more general way on the geometry need not be spherical. A striking example is provided by biology; the fluid membranes in our cells are described with uncanny accuracy, on optical scales, by the Canham-Helfrich (or Willmore) energy quadratic in the mean curvature of the membrane surface [2, 3]. In addition to the volume the area is also fixed. There may also be other constraints. Under these circumstances, determining the equilibrium shape, never mind addressing questions of stability, becomes a sutble problem. We know, however, thanks to a series of landmark numerical studies 
in the nineties that very different equilibrium shapes may be consistent with given values of these constraints and that large changes in shape may be brought about by tweaking the constraints slightly. This work is nicely summarized in references [4, 5] and [6]. Representative examples of recent work are provided by [7] and [8]. Our current theoretical framework, by constrast, remains woefully undeveloped. Even if we artificially limit ourselves to axially symmetric configurations, where it is possible to boast a level of analytical control, there is a significant discrepancy between the wealth of numerical data that has accumulated and our current ability to interpret the patterns within it - even qualitatively - on the basis of the underlying model. Some new element needs to be introduced into our theoretical framework if this imbalance is to be set right.

It is well known that is it possible to describe the equilibrium shape of the surface in terms of the conservation of a stress tensor. This problem was first approached, a la Gibbs, thermodynamically [9, 10]; such an approach, however, never really exploits the fact that the energy depends only on geometrical degrees of freedom; when the scaffolding is removed, what is left is geometry. The appropriate setting has been described in [11, 12]. Recently, Lomholt and Miao have shown how the two approaches can be reconciled [13]. From the geometrical point of view, the existence of the conservation law is a consequence of the fact that the free energy is invariant under translations. Noether's theorem identifies the stress as the conserved current associated with this invariance. Furthermore, the invariance of the geometrical freeenergy under reparametrizations of the surface implies that the stress is determined completely by the surface curvature and its derivatives. Knowledge of the local membrane geometry will always be sufficient to reconstruct the stress underpinning it. This contrasts with the behavior in an elastic solid where the stress is proportional to first derivatives and the geometry itself is not always enough to determine the state of stress [14].

Internal constraints on the geometry will add stress to the membrane. In the case of a fluid membrane, as we have mentioned, the area is fixed; the two layers of a bilayer may also have different areas. The latter asymmetry is captured by a constraint or a penalty on the total mean extrinsic curvature of the surface [15]. These constraints are accommodated using Lagrange multipliers.

Whereas surface constraints add to the stress due to bending energy, a constraint on the enclosed volume acts as an external force on the membrane. This is the well known Laplace pressure counteracting the tension in a soap bubble which acts normally to the surface. In this note, it will be shown how to incorporate this external pressure into a divergence-free effective surface stress. This makes it possible to treat the volume constraint on an equal footing with its surface counterparts. Geometrically, this is because the surface normal along which the Laplace force acts can always be expressed as a surface divergence: a fact which is itself a direct consequence of the translation invariance of volumes. Curiously, the translational invariance of the stress gets broken in the construction; the effective stress depends explicitly on the position.

The interesting point is that this unprepossessing mathematical identity permits us to look at the equilibrium in a new light. We first examine a model describing cylindrical geometries in terms of this effective stress tensor. The cross-section of a cylinder perpendicular to its axis is a closed planar loop; the free-energy on this geometry describes one of Euler's elastica subject 
to a constraint on the enclosed area on the plane. This system was studied in some detail in [16] where it was dubbed, with a nod to Euler, Elastica Hypoarealis. While it is well known that the Euler-Lagrange equation can be integrated to provide a quadrature for the curvature, this system exhibits an additional level of integrability which is not evident in its Euler-Lagrange equation: the curvature at any point detemines completely the geometry of the loop at that point. Such an identity is surprising because one would expect to require two integrations of the curvature to reconstruct the corresponding loop whereas none is needed. In this paper, it will be shown that this is no accident. In fact, it is because the conservation law can be integrated: modulo a translation centering the loop at the origin, the effective stress vanishes everywhere on the loop. The identity between curvature and position pointed out in [16] follows as an immediate consequence. Given the curvature, it is possible to trace the loop. One has not, of course, integrated the shape equation: one still needs to integrate the quadrature for the curvature as a function of arc-length in terms of elliptic integrals. But one does possess a relationship between curvature and the configuration which has sidestepped completely the need to evaluate these integrals.

The construction of the identity for a loop is loosely analogous to the Wulff construction of the equilibrium shape of a crystal from a knowledge of its anisotropic surface tension [17]. Is there an analogue for genuine two-dimensional membranes? Just as the equilibrium loop possesses a centre of 'gravity', there also will be one for a membrane. The relationship between curvature and the location with respect to this centre originates in the explicit spatial dependence of the effective stress. In a spherical equilibrium, it is simple to check that this stress vanishes when the origin is translated to the centre of the sphere. This is, in itself, a non-trivial identity concerning the balance of internal forces in the spherical equilibrium. It is, however, also simple to prove in the case of a fluid membrane that spheres are the only compact geometries possessing this property. ${ }^{1}$ One does not appear to have new to say about the shape of membranes.

There is, of course, a good reason why a straightforward analogue of the loop identities does not exist in higher dimensions. On the loop, a divergence-free (one-dimensional) effective stress is trivially constant, and this constant can be set to zero by centering the loop appropriately. In two-dimensions, however, there is enormously more freedom. The analogue of a constant vector in the plane is now a null stress, divergence-free by construction. The null stresses on a surface are in one-to-one correspondence with spatial vector fields. Furthermore, consistency with the symmetries of the fluid membrane stress tensor will place a constraint on this vector field; one finds that the admissible vector fields are the generators of area-preserving deformations of the surface. Such stresses cannot generally be eliminated by a simple translation. As a consequence, the higher dimensional generalization of the result for loops will involve null stresses. It is too early to know if this going to be tractable. However, given the enormous stakes involved (and not just for fluid membrane physics) this is certainly worth exploring.

\footnotetext{
${ }^{1}$ In the case of the Clifford torus, one can check explicitly that the effective stress is non-vanishing.
} 


\section{First order variations, Euler-Lagrange derivatives, translation in- variance and the stress}

The membrane will be described as a parametrized surface, $\xi^{a} \rightarrow \mathbf{X}\left(\xi^{a}\right)$. The $\mathbf{X}=\left(X^{1}, X^{2}, X^{3}\right)$ are three functions of two parameters $\xi^{a}, a=1,2$. The metric tensor induced on this surface is given by $g_{a b}=\mathbf{e}_{a} \cdot \mathbf{e}_{b}$; the corresponding extrinsic curvature is $K_{a b}=\mathbf{e}_{a} \cdot \partial_{b} \mathbf{n}$, where $\mathbf{e}_{a}=\partial_{a} \mathbf{X}$, $a=1,2$ are the two tangent vectors to the surface and $\mathbf{n}$ is the unit normal. $\nabla_{a}$ represents the covariant derivative compatible with $g_{a b}$. The Gauss-Weingarten equations, describing how these vectors change as one moves across the surface, are $\nabla_{a} \mathbf{e}_{b}=-K_{a b} \mathbf{n}$ and $\nabla_{a} \mathbf{n}=K_{a}{ }^{b} \mathbf{e}_{b}$. Indices are raised and lowered with the metric. When one looks at cylinders, the surface of interest will be a planar curve. Higher dimensional generalizations are also of potential interest; because it is straightforward to frame the discussion in terms of $D$-dimensional surfaces this is what will be done unless explicitly indicated otherwise. Geometrical background material is provided in [18, 19]. A nice tutotial tailored to membrane geometry is provided by reference [20].

Consider any geometrical integral of the form

$$
H[\mathbf{X}]=\int d A \mathcal{H}\left(g_{a b}, K_{a b}\right),
$$

defined on a patch of surface $\Sigma$ with a boundary $\partial \Sigma$. $H$ will represent the free energy of the surface. $\mathcal{H}$ is a function of the metric and the extrinsic curvature. Thus $H$ will be a functional of $\mathbf{X}$ and it will be invariant under reparametrizations and Euclidean motions of the ambient space. The area element induced on the surface is $d A=\sqrt{\operatorname{det} g_{a b}} d^{2} \xi$. For simplicity, gradients of $K_{a b}$ are not considered; they have, however, been considered in some detail elsewhere [21].

Recall how the stress tensor corresponding to $H$ gets constructed. This involves examining the response of the free energy to a small deformation of the surface. This deformation is described by the infinitesimal change in the embedding functions: $\mathbf{X} \rightarrow \mathbf{X}+\delta \mathbf{X}$. The response of $H$ decomposes naturally into two parts: a bulk response as well as a boundary one; the latter is a surface divergence originating in derivatives of the deformation in the bulk which have been consigned to the boundary. One finds that

$$
\begin{aligned}
\delta H= & \int d A \mathcal{E} \mathbf{n} \cdot \delta \mathbf{X}-\int d A \nabla_{a}\left[\mathbf{f}^{a} \cdot \delta \mathbf{X}\right] \\
& -\int d A \nabla_{a}\left[\mathcal{H}^{a b} \cdot \nabla_{b} \delta \mathbf{X}\right] .
\end{aligned}
$$

Here $\mathcal{E} \mathbf{n}$ is the Euler-Lagrange derivative of $H$ with respect to $\mathbf{X}$; because of reparametrization invariance of $H$, only the projection of $\delta \mathbf{X}$ onto the normal contributes to the bulk variation. $\mathbf{f}^{a}$ is the surface stress tensor associated with $H$ and $\mathcal{H}^{a b}=\partial \mathcal{H} / \partial K_{a b}$. It has been shown elsewhere [11, 12] that

$$
\mathcal{E}=-\nabla_{a} \nabla_{b} \mathcal{H}^{a b}+K_{a c} K^{c}{ }_{b} \mathcal{H}^{a b}+\mathcal{H} K
$$

and

$$
\mathbf{f}^{a}=\left(\mathcal{H}^{a c} K_{c}^{b}-\mathcal{H} g^{a b}\right) \mathbf{e}_{b}-\nabla_{b} \mathcal{H}^{a b} \mathbf{n} .
$$


When $H$ is translationally invariant it is possible to rewrite its Euler-Lagrange derivative as a surface divergence. The argument of this divergence is the stress. This follows from Eq.(2): consider the effect on the surface of a translation, $\delta \mathbf{X}=\mathbf{a}$, where $\mathbf{a}$ is a constant vector. The derivative term appearing on the second line in Eq.(2) vanishes; translational invariance, $\delta H=0$, then implies that

$$
\mathbf{a} \cdot \int d A\left[\mathcal{E} \mathbf{n}-\nabla_{a} \mathbf{f}^{a}\right]=0 .
$$

Because the region of integration itself is arbitrary, the local statement

$$
\mathcal{E} \mathbf{n}-\nabla_{a} \mathbf{f}^{a}=0
$$

follows; $\mathcal{E} \mathbf{n}$ is a surface divergence. This is a useful identity to remember.

In particular, a fluid membrane is described by the Canham-Helfrich energy

$$
H_{0}=\frac{1}{2} \int d A K^{2}
$$

where $K=g^{a b} K_{a b}$. It's mathematical properties, notably its conformal invariance, were examined long beforehand [3]. Suppose that both the area $A$ and the integrated mean curvature

$$
M=\int d A K
$$

are fixed. The free-energy describing the surface is then given by

$$
H=H_{0}+\beta\left(M-M_{0}\right)+\sigma\left(A-A_{0}\right),
$$

where $\beta$ and $\sigma$ are two Lagrange multipliers enforcing the constraints. The constants $M_{0}, A_{0}$ are the (for our purposes) irrelevant fixed valued of $M$ and $A$.

\section{Equilibrium with constrained volume}

Consider now the problem of determining the equilibrium of a closed membrane subject to a constraint on the enclosed volume. Introduce a new energy functional $\bar{H}$ related to the surface free energy $H$ by

$$
\bar{H}=H-P\left(V-V_{0}\right) .
$$

The constant Laplace pressure $P$ is a Lagrange multiplier forcing the volume to assume some fixed value, $V_{0}$. In equilibrium, the Euler-Lagrange equation $\mathcal{E}=P$ is satisfied ( $\mathcal{E}$ is the Euler-Lagrange derivative of $H$ ). Using Eq.(6), this equation can be cast as a conservation law:

$$
\nabla_{a} \mathbf{f}^{a}=P \mathbf{n} .
$$

The external pressure provides a source for the stress in the membrane. It is always possible, however, as we will now demonstrate to incorporate this particular source into an effective conserved surface stress. 


\section{Cone volume and the effective stress}

The stress was identified in section 2 by examining the boundary behaviour of the energy under deformation. One way to place the volume $V$ appearing in the constrained energy given by Eq.(10) on an equal footing with the surface invariants appearing in $H$ is to associate a notion of volume with patches of the surface. But this is easy to do: fix an origin, and construct the cone $\mathcal{C}$ with a base which consists of the patch and its apex positioned at the origin. By examining how the volume of this cone behaves under deformations of the surface patch, we will see that it is possible to associate an effective surface stress tensor with the volume term appearing in Eq.(10). It it then straightforward to recast the equilibrium condition under the external Laplace pressure in terms of a divergence-free effective surface stress.

First consider a closed surface. The volume of space it encloses is given by

$$
V=\frac{1}{D+1} \int d A \mathbf{n} \cdot \mathbf{X}
$$

This is a direct consequence of the elementary Euclidean space identity for the position vector $\mathbf{x}, \mathbf{d i v} \cdot \mathbf{x}=D+1$ and the use of the divergence theorem. While it is possible to express $V$ as a surface integral, it is not possible to express it in the form (11). Despite this, as we will now show, an analogue of Eq.(2) does hold.

Suppose that the integral on the rhs of Eq.(12) is restricted to some surface patch. It is easy to see that the integral then represents the volume of the cone $\mathcal{C}$ that was described at the beginning of this section (see [19]).

Consider now the response of the volume of this cone to a deformation of the surface $\mathbf{X} \rightarrow \mathbf{X}+\delta \mathbf{X}$. This will point us towards an extremely useful identity for the normal vector. One finds that

$$
\delta V=\int d A \mathbf{n} \cdot \delta \mathbf{X}-\frac{1}{D+1} \int d A \nabla_{a}\left(\mathbf{f}_{0}^{a} \cdot \delta \mathbf{X}\right)
$$

where

$$
\mathbf{f}_{0}^{a}=\left(\mathbf{e}^{a} \cdot \mathbf{X}\right) \mathbf{n}-(\mathbf{n} \cdot \mathbf{X}) \mathbf{e}^{a}=\mathbf{X} \times\left(\mathbf{n} \times \mathbf{e}^{a}\right) .
$$

The second expression appearing on the rhs of Eq.(14) is valid for two-dimensional surfaces. The derivation is straightforward; the details are provided for the interested reader in an appendix. In particular, the well known result that the Euler-Lagrange derivative of volume with respect to $\mathbf{X}$ is $\mathbf{n}$ is reproduced. On a closed surface, the second term in Eq.(13) vanishes so that the familiar area times normal displacement formula for the shell volume $\delta V$ follows.

The stress-like object $\mathbf{f}_{0}^{a}$ we have introduced has a very nice property: its surface divergence is proportional to $\mathbf{n}$ :

$$
\mathbf{n}=\frac{1}{D} \nabla_{a} \mathbf{f}_{0}^{a} .
$$

Curiously, the fact that $\mathbf{n}$ is a surface divergence appears to have gone unnoticed. The value of Eq.(15) is that it provides a relationship analogous to Eq.(6) for the volume with $\mathcal{E}=1$ : $\mathbf{f}_{0}^{a} / D$ is identified as the effective surface stress associated with the volume term in the energy. The derivation of Eq.(15) is elementary: note that the curvature terms, which originate in the 
Gauss-Weingarten equations for the surface, cancel when the two terms are summed. This is consistent with the fact that the normal depends only on first derivatives of $\mathbf{X}$.

A consequence of this identification is that the Laplace force on a patch of surface can be cast as a closed line integral along its boundary:

$$
P \int d A \mathbf{n}=\frac{P}{2} \oint d s(\mathbf{X} \times \mathbf{t}) .
$$

Here $\mathbf{t}$ is tangent to the boundary curve and $s$ is arc-length. ${ }^{2}$ In this form, it is clear that the Laplace force on the patch depends only on the boundary geometry, a fact that is not at all obvious when cast as an integral over the patch.

Note that the effective stress is not $\mathbf{f}_{0}^{a} /(D+1)$ as a casual comparison of Eq.(13) with Eq.(2) might suggest; this is because the cone volume, unlike the volume of a closed surface, is not translationally invariant if the apex of the cone is fixed. Indeed under a constant translation $\mathbf{a}, \mathbf{X} \rightarrow \mathbf{X}+\mathbf{a}$, and it is clear from Eq.(12) that

$$
\delta V=\frac{1}{D+1} \mathbf{a} \cdot \int d A \mathbf{n}
$$

a result consistent with Eq.(13) with the replacement of $\delta \mathbf{X}$ by $\mathbf{a}$.

The structure of the boundary term in Eq.(13) is of interest. On a two-dimensional surface, it is possible to cast this equation in the form

$$
\delta V=\int d A \mathbf{n} \cdot \delta \mathbf{X}+\frac{1}{3} \oint d s(\mathbf{t} \times \mathbf{X}) \cdot \delta \mathbf{X} .
$$

In this form, it is clear that the boundary term depends only on the boundary curve and its deformation; in particular, it is independent of what the patch gets up to in its interior.

\section{$5 \quad$ Laplace pressure as equivalent surface stress}

The identification (15) makes it possible to recast the equilibrium condition under an external Laplace pressure in terms of a divergence-free effective surface stress. Introduce the effective stress tensor,

$$
\overline{\mathbf{f}}^{a}=\mathbf{f}^{a}-\frac{P}{D} \mathbf{f}_{0}^{a} .
$$

where $\mathbf{f}_{0}^{a}$ is given by Eq.(14). Using Eq.(15), it is possible to recast the equilibrium condition Eq.(11) in the form

$$
\nabla_{a} \overline{\mathbf{f}}^{a}=0 .
$$

$\overline{\mathbf{f}}^{a}$ unlike $\mathbf{f}^{a}$ is divergence-free in equilibrium. In an isolated membrane, it follows from Eq.(20) that

$$
\oint d s l_{a} \overline{\mathbf{f}}^{a}=0
$$

\footnotetext{
${ }^{2}$ We have used the fact that for right-handed triad $\{\mathbf{l}, \mathbf{t}, \mathbf{n}\}, \mathbf{t}=\mathbf{n} \times \mathbf{l}$, where $\mathbf{l}=l^{a} \mathbf{e}_{a}$ is its outward pointing normal to the curve lying on the surface. Any two orthonormal vectors specify a third; in particular, the two normals $\mathbf{n}$ and $\mathbf{l}$ to the curve fix its tangent.
} 
along any contractible curve which lies on the surface. With the original stress tensor, the corresponding integral depends on the behaviour of the normal vector throughout the enclosed patch. Using the effective stress tensor, the volume constraint is treated like the other surface constraints.

If an external force acts on the patch of membrane enclosed by the curve, the line integral appearing in Eq.(21) will determine this force. In this context, it is often possible to deform the contour appropriately to take advantage of any symmetry the configuration might possess. This approach was developed in [22] to examine surface mediated interactions. Its generalization to incorporate a pressure difference across the membrane is also possible.

The effective stress $\overline{\mathbf{f}}^{a}$ has one very striking feature we have yet to comment upon. Unlike $\mathbf{f}^{a}, \overline{\mathbf{f}}^{a}$ is not translationally invariant. Its origin is the explicit $\mathbf{X}$ dependence appearing in $\mathbf{f}_{0}^{a}$ given by Eq.(14). Under a translation $\mathbf{X} \rightarrow \mathbf{X}+\mathbf{a}$, one finds that $\mathbf{f}_{0}^{a} \rightarrow \mathbf{f}_{0}^{a}+\mathbf{h}_{0}^{a}$, where

$$
\mathbf{h}_{0}^{a}=\mathbf{a} \times\left(\mathbf{n} \times \mathbf{e}^{a}\right) .
$$

This shift should leave no physical trace. In the next section, this issue will be examined in the appropriate setting.

\section{$6 \quad$ Null stresses}

It is clear that there is an inherent ambiguity in the definition of any divergence-free effective stress $\overline{\mathbf{f}}^{a}$; this is because one is always free to add to $\overline{\mathbf{f}}^{a}$ any stress of the form

$$
\mathbf{h}^{a}=\nabla_{b} \mathbf{A}^{a b},
$$

involving an antisymmetric potential $\mathbf{A}^{a b}=-\mathbf{A}^{b a}$. (In the case of a curve, this ambiguity degenerates into the freedom to add a constant vector.) It is straightforward to show that for $\mathbf{h}^{a}$ constructed according to Eq.(23) , one has $\nabla_{a} \mathbf{h}^{a}=0$ or $\mathbf{h}^{a}$ is conserved. We will refer to such an $\mathbf{h}^{a}$ as a null stress. In the language of differential forms $\mathbf{h}^{a}$ is exact [23].

On a two-dimensional surface, the anti-symmetric potential $\mathbf{A}^{a b}$ factorizes: it must be a product of the two-dimensional Levi-Civita anti-symmetric tensor $\epsilon^{a b}$ and a space vector potential $\mathbf{A}: \mathbf{A}^{a b}=\epsilon^{a b} \mathbf{A}$. Because $\epsilon^{a b}$ is covariantly conserved $\left(\nabla_{a} \epsilon_{b c}=0\right)$, it is possible to express the corresponding null stress in the simple form [11]

$$
\mathbf{h}^{a}=\epsilon^{a b} \nabla_{b} \mathbf{A} .
$$

How various ambiguities in the definition of $\mathbf{f}^{a}$ get captured in $\mathbf{h}^{a}$ are discussed very nicely in [13].

Suppose now that we add an $\mathbf{h}^{a}$ constructed this way to the stress; its contribution to the force on any patch is given by the line integral of $l_{a} \mathbf{h}^{a}$ along the closed boundary curve. Because $\mathbf{h}^{a}$ is exact, this integral vanishes. This is well known. To see it explicitly when $D=2$, express $\epsilon^{a b}$ in terms of the components of the unit tangent $\mathbf{t}=t^{a} \mathbf{e}_{a}$ and the normal $\mathbf{l}=l^{a} \mathbf{e}_{a}$, $\epsilon^{a b}=l^{a} t^{b}-l^{b} t^{a}$ so that

$$
\oint d s l_{a} \mathbf{h}^{a}=-\oint d s t^{a} \nabla_{a} \mathbf{A} .
$$


But $t^{a} \nabla_{a}=\partial / \partial s$ so that the closed line integral vanishes. It would appear that the notion of a null stress is not very interesting; as we will see this is not the case.

We are now in a position to comment on the behaviour of $\mathbf{f}_{0}^{a}$ under translations. It was seen that under a translation $\mathbf{X} \rightarrow \mathbf{X}+\mathbf{a}, \mathbf{f}_{0}^{a}$ transforms by $\mathbf{f}_{0}^{a} \rightarrow \mathbf{f}_{0}^{a}+\mathbf{h}_{0}^{a}$, where $\mathbf{h}_{0}^{a}$ is defined by Eq.(22). It is easy to see that $\mathbf{h}_{0}^{a}$ is, in fact, null. Indeed, when $D=2$,

$$
\mathbf{h}_{0}^{a}=\epsilon^{a b} \mathbf{a} \times \mathbf{e}_{b}=\epsilon^{a b} \nabla_{b}[\mathbf{a} \times \mathbf{X}],
$$

which is of the form (24) with the identification, $\mathbf{A}=\mathbf{a} \times \mathbf{X}$.

While a change of origin has no physical significance, the value of $\overline{\mathbf{f}}^{a}$ at a point will depend on the choice made. It may be useful to centre the origin to reflect the symmetry of the configuration. For example, in a sphere it would be perverse to place the origin anywhere but at the centre of the sphere unless there is a convincing reason to do otherwise. In the following section we will see that the explicit $\mathbf{X}$ dependence which appears in $\overline{\mathbf{f}}^{a}$ has a very important role.

\section{$7 \quad$ An identity for rigid loops enclosing a fixed area}

A strong hint that casting the conservation law in terms of an effective stress tensor might be useful is provided by examining a membrane which forms an infinite cylinder. The cross-section of the cylinder perpendicular to its axis is a closed planar loop. The free energy for the cylinder ascribes rigidity to the loop. Its length $L$ is fixed and it encloses a fixed area $A$. Consider now a curve embedded on the plane parametrized by arclength $s, \mathbf{X}=\mathbf{X}(s)$; a prime will denote a derivative with respect to $s$, so that $\mathbf{t}=\mathbf{X}^{\prime}$ is the unit tangent vector. The curvature $k$ is defined by $\mathbf{t}^{\prime}=-k \mathbf{n}$ and $\mathbf{n}^{\prime}=k \mathbf{t}$, where $\mathbf{n}$ is the unit normal. The energy penalty associated with bending is given by

$$
H_{0}=\int d s k^{2}
$$

To facilitate comparison, we will use the same normalization for the bending energy introduced in [16] (without the factor of a half), but adopt a more conventional soft-matter notation for the multipliers. Now the energy functional capturing the constraints on $L$ and $A$ is

$$
H=H_{0}+\sigma L-P A \text {. }
$$

Consider the problem of determining the equilibria of this loop. The Euler-Lagrange equation is

$$
2 k^{\prime \prime}+k^{3}-\sigma k+P=0,
$$

and it has the first integral

$$
\frac{1}{2} k^{\prime 2}+V(k)=E
$$

where

$$
V(k)=\frac{1}{8} k^{4}-\frac{\sigma}{4} k^{2}+\frac{P}{2} k
$$


and $E$ is a constant. The closure of the loop will quantize $E$. The quadrature determines the curvature $k$ implicitly as a function of $s$ in terms of elliptic functions. One would expect to require two integrations of the curvature to reconstruct the corresponding loop; remarkably, none is needed. This was shown in [16]. Here, it will be shown how this comes about using the conservation of the effective stress.

The tension in the loop is given by

$$
\mathbf{T}=\left(k^{2}-\sigma\right) \mathbf{t}-2 k^{\prime} \mathbf{n},
$$

and it satisfies

$$
\mathbf{T}^{\prime}=P \mathbf{n} .
$$

For a planar curve $(D=1)$, Eq.(15) simplifies $\mathbf{n}=[(\mathbf{X} \cdot \mathbf{t}) \mathbf{n}-(\mathbf{X} \cdot \mathbf{n}) \mathbf{t}]^{\prime}$. The equilibrium condition Eq.(33) may thus be recast as an identity for a space vector

$$
\left[\mathbf{X} \cdot \mathbf{n}+P^{-1}\left(k^{2}-\sigma\right)\right] \mathbf{t}-\left[\mathbf{X} \cdot \mathbf{t}+2 P^{-1} k^{\prime}\right] \mathbf{n}=\mathbf{C},
$$

where $\mathbf{C}$ is some constant vector along the loop. This constant is the analogue of $\mathbf{h}^{a}$ discussed earlier in the two-dimensional context. However, it is always possible to translate the origin so that $\mathbf{X} \cdot \mathbf{t}=0$ when $k^{\prime}=0$, which gives $\mathbf{C}=0$. Eq.(34) now provides two identities, one for each projection

$$
\begin{aligned}
& \mathbf{X} \cdot \mathbf{n}=P^{-1}\left(\sigma-k^{2}\right) \\
& \mathbf{X} \cdot \mathbf{t}=-2 P^{-1} k^{\prime} .
\end{aligned}
$$

The ' $\sigma$ ' identities which were identified in [16] are reproduced in a very direct way. The latter of the two identities implies that

$$
\mathbf{X}^{2}=X_{0}^{2}-4 P^{-1} k,
$$

where $X_{0}$ is a constant. Completeness of the basis vectors $\mathbf{t}$ and $\mathbf{n}$ together with Eq.(30) can now be used to express $X_{0}^{2}$ directly in terms of the constant $E$ appearing in the quadrature:

$$
X_{0}^{2}=P^{-2}\left(8 E+\sigma^{2}\right) .
$$

Once the spectrum of $E$ has been determined, the curvature at any point detemines completely the loop geometry at that point. No integrations are necessary. While it is well known that the Euler-Lagrange equation can be integrated to provide a quadrature for the curvature, this additional level of integrability is not evident in the Euler-Lagrange equation. In a beautiful paper, Joel Langer mentions the existence of such an identity somewhat obliquely as a property of the torsion-free limit of a certain integrable model for space curves [24].

It should be remarked that there is considerably greater freedom associated with an infinite or open elastic curve due to boundary conditions. No simple relationship between curvature and position is known. Nor is it expected. The configuration will depend sensitively on the boundary conditions (see, for example, [25]). Isolation is important. 


\section{Vanishing effective stress implies spherical symmetry}

It is not unreasonable to inquire if there exist identities between curvature and position in solutions to $\nabla_{a} \overline{\mathbf{f}}^{a}=0$ analogous to those for the planar elastic loop. We begin with a search for solutions satisfying the straightforward analogue to a vanishing $\mathbf{C}$ in Eq.(34), $\overline{\mathbf{f}}^{a}=0$.

Recall that translational invariance and reparametrization invariance together imply a set of integrability conditions on the components of the stress [11]. These conditions can be isolated explicitly by expanding $\mathbf{f}^{a}$ with respect to the basis $\left\{\mathbf{e}_{a}, \mathbf{n}\right\}$

$$
\mathbf{f}^{a}=f^{a b} \mathbf{e}_{b}+f^{a} \mathbf{n} .
$$

Here $f^{a b}$ is a surface tensor; $f^{a}$ is a surface vector. The projections of Eq.(6) perpendicular and parallel to the surface then read

$$
\begin{aligned}
\nabla_{a} f^{a}-K_{a b} f^{a b} & =\mathcal{E}, \\
\nabla_{a} f^{a b}+K^{b}{ }_{a} f^{a} & =0 .
\end{aligned}
$$

The (normal) Euler-Lagrange derivative is captured completely by the normal projection of the divergence; the projection process dismantles the natural surface divergence of $\mathbf{f}^{a}$.

The remaining $D$ identities (40) provide consistency conditions on the stress; they are completely independent of $\mathcal{E}$; thus, they are also independent of the equilibrium. In particular, if $H$ is a sum of terms, as it is for our constrained fluid membrane, Eq. (40) will hold for each of them separately. In Eq.(39), both the tangential stress $f^{a b}$ and the Euler-Lagrange derivative $\mathcal{E}$ act as a source for the normal stress. In Eq.(40), the normal stress provides a source for the tangential stress. Neither stress alone will generally be conserved. This coupling between normal and tangential stresses should be contrasted with the simple intrinsic conservation law for the stress $T^{a b}$ associated with a field coupling to the intrinsic surface geometry, $\nabla_{a} T^{a b}=0$.

Using Eq.(44), it is straightforward to identify the stress in a fluid membrane with an energy given by Eq.(9). One finds for the surface tensor $f^{a b}$ and the vector $f^{a}$ :

$$
\begin{aligned}
f^{a b} & =K\left(K^{a b}-\frac{K}{2} g^{a b}\right)+\beta\left(K^{a b}-K g^{a b}\right)-\sigma g^{a b} \\
f^{a} & =-\nabla^{a} K .
\end{aligned}
$$

The corresponding Euler-Lagrange derivative is given by [11, 26]

$$
\mathcal{E}=-\nabla^{2} K+\frac{1}{2} K\left(K^{2}-2 K_{a b} K^{a b}\right)+\beta \mathcal{R}+\sigma K .
$$

The intrinsic scalar curvature $\mathcal{R}$ is related to the extrinsic curvature by the contracted GaussCodazzi

$$
\mathcal{R}=K^{2}-K^{a b} K_{a b} .
$$

This setup was described in [11] and refined in [12]. Note the following points:

(1) The surface tensor $f^{a b}$ is quadratic in $g_{a b}$ and $K_{a b}$. 
(2) In a soap film, $f^{a b}=-\sigma g^{a b}$ is isotropic, with tension $\sigma$; in general, however, the mechanical surface tension is not $\sigma$. The tangential stress $f^{a b}$ depends not only on $g_{a b}$ but also on the local state of bending as characterized by $K_{a b}$.

(3) Because $f^{a b}$ depends locally on $K_{a b}$, its eigenvectors coincide with those of $K_{a b}$; the corresponding eigenvalues are quadratic functions of the principal curvatures.

(4) Umbilical points are those where the principal curvatures coincide, so that $K_{a b}=g_{a b} K / D$ (all points on a spherical membrane are umbilical). At such a point (when $D=2$ ), the bending contribution to the stress vanishes. What stresses are present are there because of competing constraints on the membrane geometry.

The components of the effective stress are given by

$$
\bar{f}^{a b}=f^{a b}+\frac{P}{D}(\mathbf{n} \cdot \mathbf{X}) g^{a b},
$$

and

$$
\bar{f}^{a}=f^{a}-\frac{P}{D}\left(\mathbf{e}^{a} \cdot \mathbf{X}\right) .
$$

Various interesting scalars can be constructed using the tangential effective stress $\bar{f}^{a b}$ given by (43). In the appendix, two of these scalars are shown to be surface divergences in equilibrium.

It is simple to show that $\overline{\mathbf{f}}^{a}=0$ in a spherical equilibrium when the origin is located at the centre of the sphere. To confirm this, note that on a $D$-dimensional sphere of radius $R$, $K=D / R$; in addition, on a sphere every point is umbilical, $K_{a b}=K g_{a b} / D$, so that the Euler-Lagrange equation $\mathcal{E}=P$ reduces to the vanishing of the following cubic polynomial in $R$ :

$$
4\left(1-\frac{2}{D}\right)+2 \beta R+\sigma R^{2}-P R^{3}=0 .
$$

This equation degenerates into a quadratic when $D=2$ because of the scale invariance of the Canham-Helfrich energy. Now, if the origin is placed at the centre of the sphere, $\mathbf{X}=R \mathbf{n}$. It is straightforward to show that $\bar{f}^{a b}=0$ coincides with the identity Eq.(45). In addition, $\bar{f}^{a}=0$ because $K$ is constant and $\mathbf{X}$ is orthogonal to the surface. Thus $\overline{\mathbf{f}}^{a}=0$. This is independent of the dimension. In particular, the scale invariance of the Canham-Helfrich energy when $D=2$ does not play a role.

The only equilibrium configurations consistent with $\overline{\mathbf{f}}^{a}=0$ are, in fact, these spheres. This follows from the vanishing of $\bar{f}^{a b}$. To see this, note that $\bar{f}^{a b}=0$ can always be cast in the form

$$
K_{a b}=F g_{a b},
$$

where

$$
F=\left[\frac{K^{2}}{2}+\beta K+\sigma-\frac{P}{D}(\mathbf{n} \cdot \mathbf{X})\right] /[K+\beta]
$$


In general, one can use completeness of the (orthonormal) principal vectors $l_{a}^{1}$ and $l_{2}^{a}$ to provide a spectral expansion for $K^{a b}$ ( $K_{1}$ and $K_{2}$ are the principal curvatures)

$$
K^{a b}=K_{1} l_{1}^{a} l_{1}^{b}+K_{2} l_{2}^{a} l_{2}^{b} .
$$

Eq.(46) then implies that the principal curvatures must coincide everywhere: $K_{1}=F=K_{2}$. What is more, they must also be constant. The simplest way to see this is to appeal to the contracted Codazzi-Mainardi equations, $\nabla_{b} K^{a b}-\nabla^{a} K=0$, which imply that $\nabla_{a} F=0$. Thus $F$ must be constant on the surface. The only solution is therefore spherical. Note that there is no corresponding constraint on the curvature of a loop (when $D=1$ the Codazzi-Mainardi equations are vacuous).

There are a few noteworthy alternative formulations of this non-existence result. Consider the following: if $\bar{f}^{a}=0$ in equilibrium, the equilibrium must be spherical and $\bar{f}^{a b}=0 .{ }^{3}$ Note that if $\bar{f}^{a}=0$, where $\bar{f}^{a}$ is given by Eq.(44), then

$$
K=C-\frac{1}{2 D} P \mathbf{X}^{2},
$$

where $C$ is a constant. This looks like a very direct analogue of Eq.(36). It turns out, however, that this equation is generally inconsisitent with its tangential counterpart; indeed the only equilibrium consistent with it is spherical. For if $\bar{f}^{a}=0$ then $\nabla_{a} \bar{f}^{a b}=0$ in equilibrium. But, on substituting the the expresssion for $\bar{f}^{a b}$ given by Eq.(43), this statement reads

$$
K^{a b}\left[\nabla_{a} K-\frac{P}{2}\left(\mathbf{e}_{a} \cdot \mathbf{X}\right)\right]=0 .
$$

If Eq.(49) is now used to express $K$ in terms of $\mathbf{X}$, there follows $\mathbf{X} \cdot \mathbf{e}_{a}=0$ or $\mathbf{X}^{2}$ is constant. Spherically symmetry is forced on us. In the next section we will explain why the naive analogue of the loop identities comes up short and suggest a generalization.

\section{Is there a generalization of the loop identities?}

It has been shown that a direct analogue of the loop identities does not exist unless the geometry is spherical. There is, however, a good reason for this failure: on the curve, the only null stress is constant; on a two-dimensional surface, however, any space vector field will generate a null stress. A more appropriate analogue is an identity of the form, $\overline{\mathbf{f}}^{a}=\mathbf{h}^{a}$, where $\mathbf{h}^{a}$ is some (hopefully) local null stress. Let us express $\mathbf{h}^{a}=h^{a b} \mathbf{e}_{a}+h^{a} \mathbf{n}$. In components,

$$
f^{a b}+\frac{P}{D}(\mathbf{n} \cdot \mathbf{X}) g^{a b}=h^{a b}
$$

and

$$
f^{a}-\frac{P}{D}\left(\mathbf{e}^{a} \cdot \mathbf{X}\right)=h^{a} .
$$

${ }^{3}$ If $\bar{f}^{a}=0$, then $K_{a b} \bar{f}^{a b}=0$ must also hold when the Euler-Lagrange equation is satisfied. 
To consistently couple $h^{a b}$ to a symmetric $f^{a b}, h^{a b}$ must also be symmetric. On one hand, this symmetry does not itself possess an invariant significance. This is because a null addition of the form (24) will not generally have a symmetric tangential component. However, the canonical $\overline{\mathbf{f}}^{a}$ for a fluid membrane does come with this symmetry so if we are to couple $\mathbf{h}^{a}$ consistently to $\mathbf{f}^{a}$, it had better also possess it.

It is simple to characterize null potentials $\mathbf{A}$ (defined in section 6) which yield symmetric $h^{a b}$. In appendix (D) we show that for $D=2, h^{a b}$ is symmetric when $\nabla_{a} \mathbf{A} \cdot \mathbf{e}^{a}=0$. This identifies the vector potential $\mathbf{A}$ with an area preserving deformation of the surface. It is straightforward to construct null stresses of this form. They are also of considerable interest in their own right leading to unexpected connections between geometrical invariants. Work in this direction will be reported elsewhere.

Whereas the symmetry of $h^{a b}$ is a necessary condition on $\mathbf{h}^{a}$, it is clearly not a sufficient one. Indeed, it is straigtforward to construct a counterexample. Suppose that $h^{a b}$ is a linear combination of $g^{a b}$ and $K^{a b}$. The null stress $\mathbf{h}^{a}$ derived from the potential $\mathbf{A}=\mathbf{X} \times \mathbf{n}$ is of this form. It is then clear from Eq.(51) that $K^{a b}$ must be proportional to $g^{a b}$. An identical argument to the one employed earlier kills all but the spherical solution. Unfortunately, we are not even close to an exhaustive list of necessary and sufficient conditions on $\mathbf{h}^{a}$. It will clearly be necessary to introduce some element of anisotropy into $\mathbf{h}^{a}$ if spherical symmetry is to be broken: its symmetry will reflect the symmetry of the configuration.

It is not, of course, obvious that Eqs.(51) and (52) are going to be tractable; they are very different from the Euler-Lagrange equations we are familiar with. What is clear is that it is worth finding out; for, if they do turn out to be tractable, the implications will be far-reaching.

\section{Discussion}

We have shown that the Laplace pressure on a closed vesicle enforcing the constraint on the enclosed volume can be incorporated into a conserved effective stress tensor. What might appear to be some mathematical sleight-of-hand does turn out to lend insight into the nature of the surface geometry.

What is perhaps most intriguing about this set-up is that it suggests that, in an isolated vesicle, it may be possible to integrate the conservation law. In a loop, where this is straightforward to do, it leads to powerful identities between the curvature and the position; control is provided over curvature without any need to solve the Euler-Lagrange equation itself explicitly. The generalization to higher-dimensional surfaces, involving the identification of the effective stress tensor with an appropriate null stress, is unfortunately anything but straightforward. The challenge is to implement it.

It should be pointed out that our results can also be cast in the language of differential forms (see, for example, [27], and in the specific context of membranes [28]). This is also likely to be useful. 


\section{Acknowledgments}

Thanks to Riccardo Capovilla, Pavel Castro-Villareal and Chryssomalis Chryssomalakos in Mexico City as well as Markus Deserno and Martin Müller in Mainz for comments. Partial support from CONACyT grant 44974-F is acknowledged.

\section{A Derivation of Eq.(13)}

To derive Eq.(13), recall that the deformation $\delta \mathbf{X}$ induces the change $\delta g_{a b}=\mathbf{e}_{a} \cdot \nabla_{b} \delta \mathbf{X}+\mathbf{e}_{b}$. $\nabla_{a} \delta \mathbf{X}$ in the metric, so that $\delta d A=d A \mathbf{e}^{a} \cdot \nabla_{a} \delta \mathbf{X}$. The deformation in the unit normal is tangential, $\delta \mathbf{n}=-\left(\mathbf{n} \cdot \nabla_{a} \delta \mathbf{X}\right) \mathbf{e}^{a}$. Thus

$$
\begin{aligned}
\delta V & =\frac{1}{D+1} \int d A\left[(\mathbf{n} \cdot \mathbf{X})\left(\mathbf{e}^{a} \cdot \nabla_{a} \delta \mathbf{X}\right)-\left(\mathbf{e}^{a} \cdot \mathbf{X}\right)\left(\mathbf{n} \cdot \nabla_{a} \delta \mathbf{X}\right)+(\mathbf{n} \cdot \delta \mathbf{X})\right] \\
& =\frac{1}{D+1} \int d A\left[(\mathbf{n} \cdot \delta \mathbf{X})-\left(\mathbf{f}_{0}^{a} \cdot \nabla_{a} \delta \mathbf{X}\right)\right] \\
& =\frac{1}{D+1} \int d A\left[(\mathbf{n} \cdot \delta \mathbf{X})+\left(\nabla_{a} \mathbf{f}_{0}^{a} \cdot \delta \mathbf{X}\right)\right]-\frac{1}{D+1} \int d A \nabla_{a}\left(\mathbf{f}_{0}^{a} \cdot \delta \mathbf{X}\right)
\end{aligned}
$$

where Eq. (14) has been used on the second line and all derivatives of $\delta \mathbf{X}$ have been collected in a divergence on the third. Now use the identity Eq.(15) on the second bulk term. Eq.(13) follows.

\section{B Identities involving $\epsilon^{a b}$}

For two-dimensional surfaces, it is useful to express $\mathrm{f}_{0}^{a}$ as a double cross product:

$$
\mathbf{f}_{0}^{a}=\mathbf{X} \times\left(\mathbf{n} \times \mathbf{e}^{a}\right)=\epsilon^{a b} \mathbf{X} \times \mathbf{e}_{b} .
$$

Here $\epsilon^{a b}$ is the two-dimensional Levi-Civita antisymmetric tensor. We have $\mathbf{e}_{a} \times \mathbf{e}_{b}=\epsilon_{a b} \mathbf{n}$, so that $\mathbf{n} \times \mathbf{e}^{a}=\epsilon^{a b} \mathbf{e}_{b}$. We note that $\epsilon^{a b} \epsilon^{c d}=g^{a c} g^{b d}-g^{a d} g^{b c}$; and, as a consequence, $\epsilon^{a c} \epsilon_{c}^{b}=-g^{a b}$.

\section{Two scalars}

We examine various scalars that can be constructed using the tangential effective stress $\bar{f}^{a b}$ given by (43).

The trace $g_{a b} \bar{f}^{a b}$ is given by

$$
g_{a b} \bar{f}^{a b}=\left(1-\frac{D}{2}\right) K^{2}+(1-D) \beta K-D \sigma+P(\mathbf{n} \cdot \mathbf{X}) .
$$


In general, $g_{a b} \bar{f}^{a b}$ will be a divergence in equilibrium. This is a consequence of the fact, observed in [11], that

$$
g_{a b} \bar{f}^{a b}=\nabla_{a}\left(\mathbf{X} \cdot \overline{\mathbf{f}}^{a}\right)
$$

when $\nabla_{a} \overline{\mathbf{f}}^{a}=0$. This identifies the argument of the divergence as $\mathbf{X} \cdot \overline{\mathbf{f}}^{a}$. Upon integration of Eq.(55) over the closed surface, there follows

$$
(2-D) H_{0}+(1-D) \beta M-D \sigma A+(D+1) P V=0 .
$$

When $D=2$, this is the well-known virial identity associated with the scaling behaviour of the energy in equilibrium [5]. We also note that the vanishing of $g_{a b} \bar{f}^{a b}$ is consistent with this identity.

In addition, $K_{a b} \bar{f}^{a b}$ is a divergence. This follows from the equilibrium condition, $\nabla_{a} \bar{f}^{a}+$ $K_{a b} \bar{f}^{a b}=0$. We have

$$
K_{a b} \bar{f}^{a b}=\frac{1}{2} K\left(K^{2}-2 \mathcal{R}\right)-\beta \mathcal{R}-\sigma K+\frac{P}{D} K(\mathbf{n} \cdot \mathbf{X}) .
$$

The contracted Gauss-Codazzi equation (42) has been used to express the quadratic $K_{a b} K^{a b}$ in terms of $\mathcal{R}$ and $K^{2}$. Using the Minkowski identity for the area [19],

$$
A=\frac{1}{D} \int d A K(\mathbf{n} \cdot \mathbf{X})
$$

one sees that the identity

$$
\int d A K_{a b} \bar{f}^{a b}=0
$$

is consistent with the integrated Euler-Lagrange equation with $\mathcal{E}$ given by Eq.(41).

\section{Symmetric $h^{a b}$ and area-preserving deformations}

Note that $h^{a b}=\mathbf{h}^{a} \cdot \mathbf{e}^{b}$ and $h^{a b}$ will be symmetric if $\epsilon_{a b} h^{a b}=0$. We now use Eq.(24) to express this condition in terms of a potential:

$$
\epsilon_{a b} h^{a b}=\nabla_{a} \mathbf{A} \cdot \mathbf{e}^{a}
$$

Thus $h^{a b}$ is symmetric when $\nabla_{a} \mathbf{A} \cdot \mathbf{e}^{a}=0$. But this condition identifies the vector potential $\mathbf{A}$ with an area preserving deformation of the surface: Under the deformation $\delta \mathbf{X}=\mathbf{A}$, it follows from the definition of $g_{a b}$ that $\delta g_{a b}=\mathbf{e}_{a} \cdot \nabla_{b} \mathbf{A}+\mathbf{e}_{b} \cdot \nabla_{a} \mathbf{A}$. The area element is proportional to $\sqrt{g}$, where $g=\operatorname{det} g_{a b}$. However,

$$
\delta g=g g^{a b} \delta g_{a b}=2 g \mathbf{e}^{a} \cdot \nabla_{a} \mathbf{A} .
$$

Thus $\delta \sqrt{g}=0$ when $\nabla_{a} \mathbf{A} \cdot \mathbf{e}^{a}=0$. This condition can be cast in terms of components tangent and normal to the surface, $\mathbf{A}=A_{\|}^{a} \mathbf{e}_{a}+A_{\perp} \mathbf{n}$. Then $\nabla_{a} \mathbf{A} \cdot \mathbf{e}^{a}=0$ is equivalent to $\nabla_{a} A_{\|}^{a}+K A_{\perp}=0$. 


\section{References}

[1] A nice discussion of this problem is provided in Kenmotsu K Surfaces with constant mean curvature (American Mathematical Society, Providence Rhode Island 2003)

[2] Canham P 1970 J. Theor. Biol. 26 61; Helfrich W 1973 Z. Naturforsch. C28 693

[3] Willmore TJ, Total Curvature in Riemannian Geometry (Chichester: Ellis Horwood, 1982)

[4] Lipowsky R and Sackmann E Structure and Dynamics of Membranes, Vol. 1 and 2 (Handbook of Biological Physics, Elsevier Science B.V. 1995)

[5] Seifert U 1997 Adv. in Phys. 4613

[6] Svetina S and Žekš B 1996 in Nonmedical Applications of Liposomes, eds. D.D. Lasic and Y. Barenholz (CRC: Boca Raton, FL)

[7] Lim G, Wortis M and Mukhopadhyay R 2002 PNAS 9916766

[8] Ziherl P and Svetina S Europhys. Lett. 200570690

[9] Evans E and Skalak R Mechanics and Thermodynamics of Biomembranes (CRC Press., Boca Raton, 1980)

[10] Kralchevsky PA Eriksson JC and Ljunggren S 1994 Adv. Coll. and Interface. Sci. 4819

[11] Capovilla R and Guven J 2002 J. Phys. A: Math. and Gen. 356233

[12] Guven J 2004 J. Phys. A: Math and Gen. 37 L313

[13] Lomholt MA and Miao L Two different descriptive approaches to the mechanics of membranes and the connection between them cond-mat/0509664

[14] Novozhilov VV Thin shell theory, Second Edition (Groningen: Noordhoff 1964)

[15] Svetina S and Žekš B 1989 Eur. Biophys. J. 17101

[16] Arreaga G, Capovilla R, Chryssomalakos C and Guven J 2002 Phys. Rev. E 65 031801; Capovilla R, Chryssomalakos C and Guven J 2002 E. Phys. J. B29 163

[17] Pimpinelli A and Villain J Physics of crystal growth (Cambridge University Press 1998)

[18] Do Carmo M Differential geometry of curves and surfaces (Prentice Hall 1976)

[19] Spivak M A Comprehensive Introduction to Differential Geometry. Vols. 1-5, Second Edition (Publish or Perish, 1979)

[20] Deserno M 2005 Lecture Notes on Differential Geometry 
[21] Guven J 2005 J. Phys. A: Math and Gen. 387943

[22] Müller M, Deserno M and Guven J Euro. Phys. Lett. 69 (2005) 482-488; Phys. Rev. E 72 (2005) 061407 'Interface mediated interactions between particles - a geometrical approach' arXiv:cond-mat/0506019

[23] Flanders H Differential forms with applications to the physical sciences (Dover Publications Inc. New York 1989)

[24] Langer J 1999 New York J. Math 525

[25] Kulic IM 2004 Statistical Mechanics of Protein Complexed and Condensed DNA (Ph.D. thesis, Max-Planck-Institut for Polymer Research, Mainz)

[26] Zhong-Can OY and Helfrich W 1987 Phys. Rev. Lett. 59 2486; 1989 Phys. Rev. A 39 5280

[27] Ivey TA and Landsberg JM Cartan for beginners (American Mathematical Society, Providence Rhode Island 2003); Griffiths P et al 2003 Exterior Differential Systems and EulerLagrange Partial Differential Equations (Chicago Lectures in Mathematics Series)

[28] Tu ZC and Ou-Yang ZC 2004 J. Phys. A: Math. Gen. 3711407 